with pibrentasvir $(\mathrm{G} / \mathrm{P})$ are approved for the treatment of chronic HCV genotype (GT) 1-6 infection. However, the efficacy and safety data of $\mathrm{G} / \mathrm{P}$ are limited in patients infected with HCV GT5 and GT6.

Methods The data analysis integrated data from 10 Phase $2 \mathrm{~b}$, 3a (registrational) and $3 \mathrm{~b}$ (post-registrational) studies. The patient population comprised adults with chronic HCV GT5 or GT6 infection and compensated liver disease (with or without cirrhosis) who were treatment-naïve or experienced with regimens containing interferon (IFN) or pegylated IFN (pegIFN) \pm ribavirin or sofosbuvir + ribavirin \pm pegIFN. Patients received 8 or 12 weeks of G/P $(300 \mathrm{mg} / 120 \mathrm{mg})$ depending on the design of the original study. Efficacy was evaluated as the rate of sustained virologic response (SVR) at post-treatment week (PTW) 12 (SVR12). HCV subtype was identified by phylogenetic analysis.

Results Data from 181 patients were included in this integrated analysis, including $56(30.9 \%)$ with HCV GT5 and 125 (69.1\%) with HCV GT6 infection. Overall, 102 (56.4\%) patients were treated for 8 weeks and 79 (43.6\%) for 12 weeks, irrespective of the presence of cirrhosis. Most patients were male (54.1\%), Asian (64.6\%), < 65 years of age (72.4\%), treatment-naïve $(87.8 \%)$, with F0-F1 fibrosis $(68 \%)$, and non-cirrhotic (non-F4, 84.5\%). Overall, 1\% (1/102) of patients on 8-week treatment and no $(0 / 76)$ patient on 12 week treatment experienced a relapse. SVR12 rates by cirrhosis status and treatment duration are shown in (Abstract IDDF2019-ABS-0211 figure 1). Treatment-emergent adverse events (AEs) were mostly mild or moderate in severity (175/ 181, 96.7\%). Most common AEs in at least $10 \%$ of patients were fatigue $(29 / 181,16 \%)$ and headache $(27 / 181,14.9 \%)$.

Conclusions HCV GT5- and GT6-infected patients without cirrhosis or with compensated cirrhosis treated with $\mathrm{G} / \mathrm{P}$ for 8 or 12 weeks achieved high rates of SVR12. There were no added efficacy benefits when treatment was extended from 8 to 12 weeks, even in patients with compensated cirrhosis. The $\mathrm{G} / \mathrm{P}$ regimen was well-tolerated.

\section{IDDF2019-ABS-0212 REAL-WORLD EFFECTIVENESS AND SAFETY OF GLECAPREVIR/PIBRENTASVIR IN ADULTS WITH CHRONIC HEPATITIS C VIRUS INFECTION: A META-ANALYSIS}

\begin{abstract}
${ }^{1}$ Markus Cornberg*, ${ }^{2}$ Francesco Negro, ${ }^{3}$ Pietro Lampertico, ${ }^{4}$ Juan Turnes, ${ }^{5}$ Michael P Curry, ${ }^{6}$ Ashley Brown, ${ }^{7}$ Heiner Wedemeyer, ${ }^{8}$ Jose A Carrion, ${ }^{9}$ Nicole Wick, ${ }^{10}$ Andreas Pangerl. ${ }^{1}$ Department of Gastroenterology, Hepatology and Endocrinology, Hannover Medical School, Hannover, Germany; 'Divisions of Gastroenterology and Hepatology and of Clinical Pathology, University Hospital, Geneva, Switzerland; 'Fondazione IRCCS Ca' Granda, Ospedale Maggiore Policlinico, University of Milan, Milan, Italy; ${ }^{4}$ Department of Gastroenterology and Hepatology, C.H.U. Pontevedra and IIS Galicia Sur, Spain; ${ }^{5}$ Beth Israel Deaconess Medical Center, Boston, MA, USA; ${ }^{6}$ Imperial College Healthcare NHS Trust, London, UK; ${ }^{7}$ University Clinic Essen, Essen, Germany; ${ }^{8}$ Liver Section, Gastroenterology Department, Hospital del Mar, IMIM (Hospital del Mar Medical Research Institute), UAB (Universitat Autonoma de Barcelona), Barcelona, Spain; ${ }^{9}$ Trio Health, La Jolla, USA; ${ }^{10}$ AbbVie Inc., North Chicago, IL, USA
\end{abstract}

\subsection{6/gutjnl-2019-IDDFabstracts.286}

Background Glecaprevir/pibrentasvir (G/P) is approved for adults infected with hepatitis $\mathrm{C}$ virus (HCV) genotypes 1-6. In clinical trials, $\mathrm{G} / \mathrm{P}$ was associated with high rates of sustained virologic response at post-treatment Week 12 (SVR12) and was well tolerated. Currently, real-world evidence (RWE) regarding $\mathrm{G} / \mathrm{P}$ use is being collected. A systematic review and meta-analysis of RWE reporting the effectiveness and safety of $\mathrm{G} / \mathrm{P}$ were undertaken.

Methods Biosis, Derwent Drug File, Embase ${ }^{\circledR}$, International Pharmaceutical Abstracts, Medline ${ }^{\circledR}$, and SciSearch databases were searched using pre-defined terms for ' $G / P$ ' and 'RWE' to identify real-world prospective/retrospective studies (1 January 2017-15 October 2018) that reported SVR12 and/or safety parameters in $\mathrm{HCV}$-infected adults $(\mathrm{N} \geq 20)$ treated with $\mathrm{G} / \mathrm{P}$. Congress presentations up to 12 November 2018 were included. Random effects meta-analysis was used to determine SVR12 and naïve pooling for adverse event (AE) rates. Intention-to-treat (ITT) SVR12 analyses included all patients dosed with G/P; modified ITT (mITT) excluded those who had nonvirologic failure.

Results 10,048 adults treated with $\mathrm{G} / \mathrm{P}$ in 16 studies were included; ITT SVR12 rates were reported in 14 studies and mITT SVR12 rates in 11 studies. SVR12 rates overall and by subgroups based on ITT and mITT populations are shown (table 1). AEs were summarised in 6 studies and reported in $12.7 \%(724 / 5685)$ of patients. Treatment discontinuations due to AEs were summarised in 5 studies and reported in $0.5 \%$ $(24 / 4508)$ of patients. The most frequent AEs were pruritus $(4.7 \% ; 126 / 2698)$, fatigue $(4.4 \% ; 146 / 3305)$, and headache $(2.7 \% ; 102 / 3759)$. SVR12 and safety data will be updated in the final presentation.

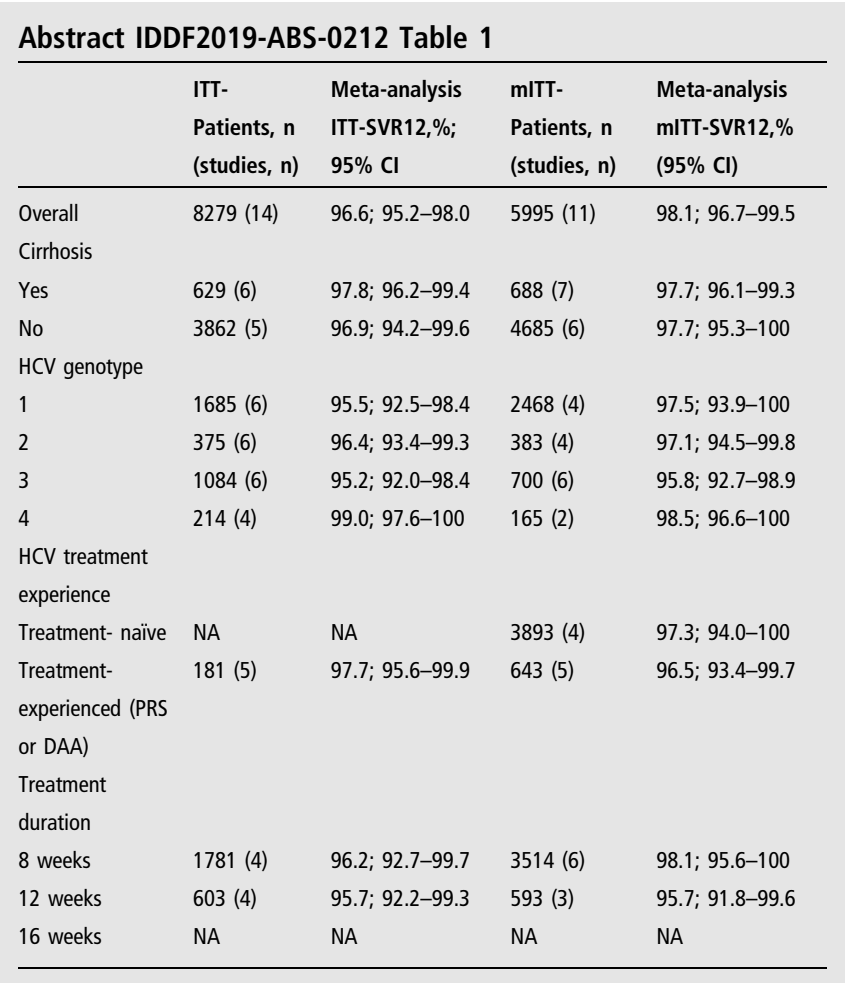

Conclusions Consistent with results observed in clinical trials, RWE indicates that G/P is a well-tolerated and highly effective pangenotypic treatment option for a broad range of $\mathrm{HCV}$ infected patients (table 1). 